\title{
Computed tomographic colonography in preoperative evaluation of colorectal tumors: a prospective study
}

\author{
Krzysztof Leksowski • Malgorzata Rudzinska • \\ Janusz Rudzinski
}

Received: 8 July 2010/ Accepted: 22 December 2010/Published online: 17 March 2011

(c) The Author(s) 2011. This article is published with open access at Springerlink.com

\begin{abstract}
Background This study aimed to assess the usefulness of computed tomographic colonography (CTC) in preoperative evaluation of colorectal tumors and the entire bowel including endoscopically inaccessible regions.

Methods Colonoscopy and CTC were performed for 49 patients. The tumor and the entire colon were assessed, and the results were compared with colonoscopy. The extraluminal findings of CTC were compared with contrast-enhanced computed tomography (CT) of the abdomen and the pelvis in 33 patients. All these patients had undergone surgery. A comparison of results for tumor node metastasis classification between CTC, CT, and histopathology was performed.

Results Exploration of the entire colon was possible for $89.8 \%$ of the patients using CTC and $49.0 \%$ of the patients using colonoscopy. Bowel cleansing was assessed as worse with CTC. In the evaluation of tumor location and morphologic type, CTC was congruent with colonoscopy.
\end{abstract}

K. Leksowski $(\bowtie)$

Department of General, Thoracic, and Vascular Surgery,

10th Military Clinical Hospital, Powstancow Warszawy 5 Str,

85-681 Bydgoszcz, Poland

e-mail: leksowski@poczta.onet.pl

K. Leksowski

Chair of Public Health Collegium Medicum in Bydgoszcz,

Nicolaus Copernicus University, Torun, Poland

M. Rudzinska

Clinical Department of Radiology, 10th Military Clinical

Hospital, Powstancow Warszawy 5 Str,

85-681 Bydgoszcz, Poland

J. Rudzinski

Clinical Department of Gastroenterology, 10th Military Clinical

Hospital, Powstancow Warszawy 5 Str,

85-681 Bydgoszcz, Poland
Colonoscopy enabled approximate tumor size and volume to be evaluated for only $59.2 \%$ (29/49) and 30.6\% (15/49) of patients, respectively, whereas CTC enabled evaluation of all $48(100.0 \%)$ visualized tumors. Wall thickening, outer contour, and suspected infiltration of surrounding tissues and organs are impossible to determine with colonoscopy but can be determined with CTC. Using CTC, two additional tumors were found proximate to occlusive masses in endoscopically inaccessible regions.

Conclusion Computed tomographic colonography is a useful method for diagnosing colorectal tumors. It allows the clinician to diagnose tumor, determine local tumor progression, and detect synchronous lesions in the large bowel including endoscopically inaccessible regions.

Keywords Colonoscopy - Colorectal neoplasms · Computed tomographic colonoscopy · Diagnostic imaging

For patients with colorectal cancer, precise preoperative staging and evaluation of the entire bowel are essential for the planning of optimal therapy [1-3]. Conventional colonoscopy, regarded as a highly sensitive and specific diagnostic method, still is considered the gold standard for detecting colorectal neoplasm [4]. However, it fails to show the entire colon in $0.6-20.0 \%$ of examinations due to technical reasons and patient intolerance. In addition, colonoscopy does not allow evaluation of extraluminal structures. Therefore, for assessment of colorectal cancer staging, other diagnostic tools are necessary. The most common tool is multidetector contrast-enhanced computed tomography (CT) of the abdomen and pelvis [5-9]. Computed tomographic colonography (CTC) has the potential to become an accepted technique for both detecting and staging colorectal cancer as well as for evaluating the entire colon in these patients. 
This study aimed to assess the usefulness of CTC in preoperative evaluation of colorectal tumors and the entire bowel.

\section{Methods}

This prospective study recruited 49 consecutive patients (31 men and 18 women, ages 24-88 years; median age 70 years) with colorectal tumors identified at conventional colonoscopy. All these patients were referred for CTC. Contrast-enhanced CT of the abdomen and pelvis was performed within a few days if required (in case of large or infiltrative tumor or malignancy in histopathologic results).

The day before the examination, each patient ingested a standard bowel preparation consisting of 4-5 1 of polyethylene glycol electrolyte solution (Fortrans; Beaufour Ipsen, Paris, France). Colonoscopy was performed by experienced endoscopists with the patient under general anesthesia or in sedation with midazolam (Dormicum, Roche, France). During the examination, tissue samples were taken from each tumor for histopathologic assessment.

The same day, CTC was performed according to a standard protocol after the colonoscopy. Patients were placed in the right lateral decubitus position on the CT table, and room air (2.0-2.5 1 or to the patient's tolerance) was insufflated through an enema tube inserted in rectum. To reduce bowel peristalsis and colonic spasm, $40 \mathrm{mg}$ of $N$-butyl-skopolamin (Buscolysin, Sopharma, Poland) was administered intravenously immediately before insufflation.

The tube was removed, and a CT scout view image was acquired to assess the degree of colonic distension. More air was insufflated if required. Helical CT scans were obtained through the entire abdomen and pelvis during a single breathhold with the patient in supine and prone positions. The CTC procedure was performed with 64-slice row CT (Siemens Sensation Cardiac 64; Siemens, Erlangen, Germany) at a collimation of $0.6 \mathrm{~mm}$, a rotation time of $0.5 \mathrm{~s}$, a reconstruction interval of $0.7 \mathrm{~mm}$, pitch of 1.4 , $120 \mathrm{kV}$, and 50 or $30 \mathrm{~mA}$ with the patient in the supine or prone position adequately. Using these parameters $\mathrm{CTDI}_{v o l}$ amounted to $3.9 \mathrm{mGy}$ in the supine position and $2.3 \mathrm{mGy}$ in the prone position, and the effective dose for the average man or woman amounted to 2.40 or $3.71 \mathrm{mSv}$ in the supine position and 1.44 or $2.23 \mathrm{mSv}$ in the prone position. Acquired CT data were transferred to a workstation equipped with software (Software Version syngo CT 2006A, Siemens) adjusted to CTC evaluation.

All CTC images were interpreted by the same radiologist, who was blinded to specific details including the location and size of the tumor and other colorectal findings. Three-dimensional (3D) endoluminal "fly-through" views were evaluated primarily using both retrograde (rectum to cecum) and antegrade (cecum to rectum) navigations. Twodimensional multiplanar reformatted images were assessed to verify the morphologic features of the lesions. Threedimensional volume-rendering technique reconstructions were used to determine lesion locations. Both supine and prone image data sets were evaluated.

The presence, location, size, and morphologic features of colorectal tumors were assessed using both colonoscopy and CTC. Tumor location was determined by reference to the following eight colonic segments: cecum, ascending colon, hepatic flexure, transverse colon, splenic flexure, descending colon, sigmoid colon, and rectum. Size was evaluated in colonoscopy by reference to biopsy forceps and in CTC by measurement on multiplanar reconstructed images (three perpendicular dimensions). Approximate volume was calculated if three dimensions were available. Morphologically, tumors were assessed as polypoid or infiltrating. Additionally, in CTC outer contour, infiltration of surrounding tissues and organs were evaluated.

The adequacy of bowel cleansing was evaluated with both colonoscopy and CTC using a 5-point scale ranging from 1 (inadequate bowel preparation) to 5 (optimal bowel preparation). Points 4 and 5 point denoted good bowel cleansing. For cleansing evaluation, the bowel was divided into 10 segments including the 8 segments described earlier and 2 additional segments, namely, the descendent colon/ sigmoid junction and the sigmoid colon/rectal junction. Colon distension was determined and described for each of the 10 segments as well-distended, inadequately-distended, or collapsed. Each patient subjectively assessed tolerance of colonoscopy and CTC using a 5-point scale ranging from 1 (total intolerance) to 5 (very good tolerance).

The CTC results were compared with the colonoscopic findings. The extraluminal findings were correlated with the contrast-enhanced CT of the abdomen and pelvis.

The study protocol was approved by the Research and Ethics Committee of the Medical University, and written informed consent was obtained from all the participants in the study.

Statistical analysis

Statistical analyses were conducted with descriptive statistics (means and percentages), two fraction tests, Snedecor's $F$ test, the $Z$-test, Student's $t$-test, and Cochran-Cox's test. All $p$ values less than 0.05 were considered significant.

\section{Results}

Table 1 presents the comparison between colonoscopy and CTC. For 25 patients $(51.1 \%)$, colonoscopy was incomplete because of obstructive or occlusive masses. Using 
CTC, visualization of the entire colon was obtained for 44 patients $(89.8 \%)$. Five patients $(10.2 \%)$ had incomplete examination with CTC due to inadequate bowel distension caused by difficulties in air holding or occlusive tumor. For one patient, CTC failed due to a completely collapsed colon and no possibility of its evaluation. Therefore, 48 patients in this group were included in the analysis.

Bowel cleansing was assessed as worse using CTC. In the supine position, the worst cleansed bowel segments were the rectum and the sigmoid colon/rectal junction. However, the same segments were well visualized in the prone position because residual fluid and feces moved to the sigmoid and descending colon. The worst cleansed and distended segment in prone position was the transverse colon, which was well visualized in the supine position. Bowel cleansing proximate and distal to the obstructive tumor showed no significant difference based on positioning of the patient.

The CTC method correctly identified 48 (98.0\%) of 49 tumors. One tumor $(2.0 \%)$ was not shown because of nondiagnostic examination. The remaining tumors were well visualized including four incomplete CTCs.

For evaluation of tumor location and morphologic type, CTC was congruent with colonoscopy. Small divergences in tumor location occurred for adjacent segments (e.g. splenic flexure and the proximal part of the descending colon). Tumors were predominantly infiltrating (61.2\%). All occlusive tumors were of this type.

Using colonoscopy, evaluation of approximate tumor size and volume was possible for only $59.2 \%$ (29/49) and $30.6 \%(15 / 49)$ of patients, respectively, in assessment of nonocclusive, small, and medium-sized tumors. Using CTC, it was possible to evaluate all 48 visualized tumors (100.0\%). Determination of wall thickening, outer contour, and suspected infiltration of surrounding tissues and organs was impossible using colonoscopy but possible using CTC. With CTC, two additional tumors were found proximate to occlusive masses in endoscopically inaccessible regions. In one patient with occlusive tumor in the transverse colon, the second large lesion $(7.0 \times 6.5 \times 6.0 \mathrm{~cm})$ was found in the cecum and confirmed in contrast-enhanced CT of the abdomen. In another patient, a round mass with a diameter of $3 \mathrm{~cm}$ proximal to obstructive lesion was depicted using CTC. However, it was not found in the control colonoscopy (the first colonoscopy was incomplete). It probably was formed in the stool mass, imitating tumor.

The histopathologic results of tissue samples taken during colonoscopy showed 42 adenocarcinomas, 3 adenomas, 1 adenoma with focal cancer, and 3 nonspecific cells. Four synchronous tumors $(8.2 \%)$, shown with both colonoscopy and CTC, were benign (large adenomatous polyps).

Among 43 patients with malignant tumors, 10 did not agree to undergo surgery. For all the remaining 33 patients (67.3\%), contrast-enhanced CT of the abdomen and pelvis was compared with CTC, and results of the comparison are presented in Table 2. All these patients underwent surgery, and for all of them, adenocarcinoma was confirmed at the postoperative histopathologic examination. Table 3 compares the results of tumor node metastasis (TNM) classification using CTC, CT, and histopathology. No complications occurred with either colonoscopy or CTC.

\section{Discussion}

The effectiveness of CTC in detecting colorectal tumors has been confirmed in several studies. The reported sensitivity is $100 \%$ or approximately $100 \%$ [7, 10-14].

An accurate tumor location may influence surgical performance including location of the incision, placement of laparoscopic ports, extent of the resection, and even stoma-site planning. With colonoscopy, anatomic variation and the absence of fixed internal landmarks make it
Table 1 Results of comparison between colonoscopy and computed tomographic colonography (CTC)

\begin{tabular}{lccc}
\hline & Colonoscopy $n(\%)$ & CTC $n(\%)$ & $p$ value \\
\hline Complete examination & $24(48.9)$ & $44(89.8)$ & $<0.001$ \\
Incomplete examination & $25(51.1)$ & $5(10.2)$ & $<0.001$ \\
Mean bowel cleansing (range) & $4.66(3.70-5.00)$ & $4.29(2.80-5.0)$ & 0.001 \\
Occlusive tumor & $25 / 49(51.0)$ & $3 / 48(6.2)$ & $<0.0001$ \\
Morphologic tumor type & & & \\
$\quad$ Infiltrating & $30 / 49(61.2)$ & $30 / 48(62.5)$ & 1.00 \\
Polypoid & $19 / 49(38.8)$ & $18 / 48(37.5)$ & 0.83 \\
Tumor size evaluation (at least 1 diameter) & $29 / 49(59.2)$ & $48 / 48(100.0)$ & $<0.0001$ \\
Wall-thickening evaluation & $0 / 49(0.0)$ & $48 / 48(100.0)$ & $<0.0001$ \\
Synchronic tumors & $4(8.2)$ & $6(12.5)$ & - \\
Untypical colon topography & $5 / 49(10.2)$ & $24 / 49(49.0)$ & $<0.0001$ \\
Mean tolerance of examination (range) & $3.51(2.0-5.0)$ & $4.23(3.0-5.0)$ & $<0.0001$ \\
\hline
\end{tabular}


Table 2 Comparison between computed tomographic (CT) colonography (CTC) and contrast-enhanced computed tomography (CT) of the abdomen

\begin{tabular}{|c|c|c|c|}
\hline & CTC $(n=33)$ & $\mathrm{CT}(n=33)$ & $p$ value \\
\hline Fat tissue infiltration at the site of tumor, $n(\%)$ & $26(78.8)$ & $20(60.6)$ & 0.10 \\
\hline Infiltration of surrounding organs at the site of tumor, $n(\%)$ & $12(36.4)$ & $9(27.3)$ & 0.43 \\
\hline Presence of enlarged ( $\geq 10 \mathrm{~mm}$ ) abdominal and pelvic lymph nodes, $n(\%)$ & $11(33.3)$ & $13(39.4)$ & 0.61 \\
\hline
\end{tabular}

Table 3 A comparison of tumor node metastases (TNM)

\begin{tabular}{llll}
\hline & $\begin{array}{l}\text { CT colonography } \\
(n=33)\end{array}$ & $\begin{array}{l}\text { Contrast-enhanced } \\
\text { CT }(n=33)\end{array}$ & $\begin{array}{l}\text { Histopathology } \\
(n=33)\end{array}$ \\
\hline ST2,n(\%) & $7(21.2)$ & $13(39.4)$ & - \\
$\mathrm{T} 3, n(\%)$ & $14(42.4)$ & $11(33.3)$ & $33(100.0)$ \\
$\mathrm{T} 4, n(\%)$ & $12(36.4)$ & $9(27.3)$ & - \\
$\mathrm{N}>1.0 \mathrm{~cm}, n(\%)$ & $11(33.3)$ & $13(39.4)$ & - \\
$\mathrm{N}, n(\%)$ & - & - & $\mathrm{N} 0-19(57.5)$ \\
& & & $\mathrm{N} 1-10(30.3)$ \\
$\mathrm{M}, n(\%)$ & - & - & $\mathrm{N} 2-4(12.2)$ \\
\hline
\end{tabular}

$C T$ computed tomography

With standard CTC, routine use of contrast enhancement is not necessary. Therefore, this study aimed to assess bowel not extracolonic organs [18-20]. In our study, we used standard CTC without contrast administration, and the extracolonic findings were compared with contrastenhanced CT of the abdomen and pelvis. Although our results were satisfactory, we believe an indication exists for patients with colorectal cancer to undergo contrastenhanced CTC because it has the potential advantage of providing images not only of the bowel but also of extracolonic tissues and the liver in one setting. Therefore, additional complementary examination may be unnecessary [9, 13, 19-21].

Preoperative evaluation of the entire colon in patients with colorectal cancer is widely recommended due to the high prevalence of synchronous tumors. It is estimated that 1.5-9.0\% of patients with colorectal carcinoma have a coexistent synchronous cancer and that $27-55 \%$ have coexistent adenomatous polyps [21-24]. Although conventional colonoscopy is regarded as the gold standard for evaluating the colon before surgery, studies have shown that the entire colon is visualized in only $42-60 \%$ of patients with colon cancer, mainly because of occlusive masses [25, 26].

Until recently, double-contrast barium enema was performed for preoperative evaluation of the proximal colon. However, this method may be limited by poor coating of the proximal bowel, low accuracy in detecting polyps, and the problem of residual barium during surgery [21, 25]. Currently, CTC is considered preferable to barium enema 
for examination. Satisfactory effectiveness of CTC for evaluating the entire colon after incomplete colonoscopy has been reported by several authors and proved in our study as well [7, 17, 21, 27, 28].

With CTC, it is not possible to avoid false diagnoses. Improvements in $\mathrm{CT}$ technology and acquired experience of radiologists help to reduce false results. It generally is accepted that a well-prepared colon, which is mandatory for optimal visualization and interpretation, is clean, welldistended, and "dry" [10, 13, 15, 20, 29]. Poor preparation is the main reason for missed or false recognized colorectal lesions [29-31].

In our study, poor bowel cleansing significantly influenced the evaluation time and the interpretative doubts. Insufficient colon distension was the reason of incomplete CTC because evaluation of a collapsed colon is not possible. We did not notice a difference in bowel cleansing and distension between bowel proximal to an occlusive tumor and bowel distal to it. Luminal narrowing and spasm associated with diverticular disease as well surrounding tissue infiltration in acute diverticulitis can lead to difficulties in interpretation because these can mimic neoplastic infiltration [15]. In our study, we encountered such problems in two patients.

Our study findings show that CTC is a useful method for the diagnosis of advanced colorectal tumors. It allows diagnosis of tumor, determination of local tumor progression, and detection of synchronous lesions in the large bowel, even in endoscopically inaccessible regions. On the basis of our patients, we cannot evaluate whether CTC is a useful method for low disease stages as well. Nevertheless, some authors have shown that contrast-enhanced CTC is accurate in the preoperative assessment of tumors staged lower than T2 [9].

To be sure, CT colonography cannot completely replace colonoscopy, but it has the potential to become a complementary or alternative method for colorectal tumor diagnosis. Further multicenter randomized studies are necessary to determine the role of CTC in colorectal tumor staging and to establish the optimal CT colonography methods.

Disclosures Krzysztof Leksowski, Malgorzata Rudzinska, and Janusz Rudzinski have no conflicts of interest or financial ties to disclose.

Open Access This article is distributed under the terms of the Creative Commons Attribution Noncommercial License which permits any noncommercial use, distribution, and reproduction in any medium, provided the original author(s) and source are credited.

\section{References}

1. McCormick JT, Gregorcyk SG (2006) Preoperative evaluation of colorectal cancer. Surg Oncol Clin North Am 15:39-49
2. Tamandl D, Herberger B, Gruenberger B, Schoppmann SF, Puhalla H, Schindl M, Schima W, Jakesz R, Gruenberger T (2008) Adequate preoperative staging rarely leads to a change of intraoperative strategy in patients undergoing surgery for colorectal cancer liver metastases. Surgery 143:648-657

3. Nesbitt C, Glendinning RJ, Byrne C, Poston GJ (2007) Factors that influence treatment strategies in advanced colorectal cancer. Eur J Surg Oncol 33:S88-S94

4. Smith RA, Cokkinides V, Brawley OW (2008) Cancer screening in the United States, 2008: a review of current American Cancer Society guidelines and cancer screening issues. CA Cancer J Clin 58:161-179

5. Hanson ME, Pickhardt PJ, Kim DH, Pfau PR (2007) Anatomic factors predictive of incomplete colonoscopy based on findings at CT colonography. AJR Am J Roentgenol 189:774-779

6. Marshall JB, Barthel JS (1993) The frequency of total colonoscopy and terminal ileal intubation in the 1990s. Gastrointes Endosc 39:518-520

7. Copel L, Sosna J, Kruskal JB, Raptopoulos V, Farrell RJ, Morrin MM (2007) CT colonography in 546 patients with incomplete colonoscopy. Radiology 244:471-478

8. van Gelder RE, Nio CY, Florie J, Bartelsman JF, Snel P, de Jager SW, Van Deventer SJ, Lameris JS, Bossuyt PM, Stoker J (2004) Computed tomographic colonography compared with colonoscopy in patients at increased risk for colorectal cancer. Gastroenterology 127:41-48

9. Filippone A, Ambrosini R, Fuschi M, Marinelli T, Genovesi D, Bonomo L (2004) Preoperative $\mathrm{T}$ and $\mathrm{N}$ staging of colorectal cancer: accuracy of contrast-enhanced multidetector row CT colonography: initial experience. Radiology 231:83-90

10. Pickhardt PJ, Choi JR, Hwang I, Butler JA, Puckett ML, Hildebrandt HA, Wong RK, Nugent PA, Mysliwiec PA, Schindler WR (2003) Computed tomographic virtual colonoscopy to screen for colorectal neoplasia in asymptomatic adults. N Engl J Med 349:2191-2200

11. Mainenti PP, Cirillo LC, Camera L, Persico F, Cantalupo T, Pace L, De Palma GD, Persico G, Salvatore M (2006) Accuracy of single phase contrast enhanced multidetector CT colonography in the preoperative staging of colorectal cancer. Eur J Radiol 60:453-459

12. Kim JH, Kim WH, Kim TI, Kim NK, Lee KY, Kim MJ, Kim KW (2007) Incomplete colonoscopy in patients with occlusive colorectal cancer: usefulness of CT colonography according to tumor location. Yonsei Med J 48:934-941

13. Chung DJ, Huh KC, Choi WJ, Kim JK (2005) CT colonography using 16-MDCT in the evaluation of colorectal cancer. Am J Roentgenol 184:98-103

14. Fletcher JG, Johnson CD, Krueger WR, Ahlquist DA, Nelson H, Ilstrup D, Harmsen WS, Corcoran KE (2002) Contrast-enhanced CT colonography in recurrent colorectal carcinoma: feasibility of simultaneous evaluation for metastatic disease, local recurrence, and metachronous neoplasia in colorectal carcinoma. Am J Roentgenol 178:283-290

15. Smith CS, Fenlon HM (2002) Virtual colonoscopy. Best Pract Res Clin Gastroenterol 16:219-236

16. Amin Z, Boulos PB, Lees WR (1996) Technical report: spiral CT pneumocolon for suspected colonic neoplasms. Clin Radiol 51:56-61

17. Neri E, Giusti P, Battolla L, Vagli P, Boraschi P, Lencioni R, Caramella D, Bartolozzi C (2002) Colorectal cancer: role of CT colonography in preoperative evaluation after incomplete colonoscopy. Radiology 223:615-619

18. Barish MA, Soto JA, Ferrucci JT (2005) Consensus on current clinical practice of virtual colonoscopy. AJR Am J Roentgenol 184:786-792

19. Morrin MM, Farrell RJ, Kruskal JB, Reynolds K, McGee JB, Raptopoulos V (2000) Utility of intravenously administered contrast material at CT colonography. Radiology 217:765-771 
20. Hoppe H, Netzer P, Spreng A, Quattropani C, Mattich J, Dinkel HP (2004) Prospective comparison of contrast-enhanced CT colonography and conventional colonoscopy for detection of colorectal neoplasms in a single institutional study using secondlook colonoscopy with discrepant results. Am J Gastroenterol 99:1924-1935

21. Fenlon HM, McAneny DB, Nunes DP, Clarke PD, Ferrucci JT (1999) Occlusive colon carcinoma: virtual colonoscopy in the preoperative evaluation of the proximal colon. Radiology 210:423-428

22. Langevin JM, Nivatvongs S (1984) The true incidence of synchronous cancer of the large bowel: a prospective study. Am J Surg 147:330-333

23. Chen HS, Sheen-Chen SM (2000) Synchronous and "early" metachronous colorectal adenocarcinoma: analysis of prognosis and current trends. Dis Colon Rectum 43:1093-1099

24. Fante R, Roncucci L, Di G et al (1996) Frequency and clinical features of multiple tumors of the large bowel in the general population and in patients with hereditary colorectal carcinoma. Cancer 77:2013-2021

25. Tate JJ, Rawlinson J, Royle GT, Brunton FJ, Taylor I (1988) Preoperative or postoperative colonic examination for synchronous lesions in colorectal cancer. Br J Surg 75:1016-1018
26. Bat L, Neumann G, Shemesh E (1985) The association of synchronous neoplasms with occluding colorectal cancer. Dis Colon Rectum 28:149-151

27. Huh KC, Yoon HJ, Lee TH (2005) The usefulness of preoperative CT colonography to evaluate the proximal colon. Gastrointest Endose 61:W1110

28. American Gastroenterological Association Institute (2006) Position of the American Gastroenterological Association (AGA) Institute on computed tomographic colonography. Gastroenterology 131:1627-1628

29. Doshi T, Rusinak D, Halvorsen RA, Rockey DC, Suzuki K, Dachman AH (2007) CT colonography: false-negative interpretations. Radiology 244:165-173

30. Park SH, Ha HK, Kim MJ, Kim KW, Kim AY, Yang DH, Lee MG, Kim PN, Shin YM, Yang SK, Myung SJ, Min YI (2005) False-negative results at multi-detector row CT colonography: multivariate analysis of causes for missed lesions. Radiology 235:495-502

31. Mang T, Maier A, Plank C, Mueller-Mang C, Herold C, Schima W (2007) Pitfalls in multidetector row CT colonography: a systematic approach. Radiographics 27:431-454 